\title{
An Energy-Balancing and Spectrum-Aware Clustering Algorithm for Cognitive Radio Sensor Network*
}

\author{
Xuehan Chen \\ School of Software, Central South \\ University \\ Changsha, Hunan, China 410075 \\ xhss1991@163.com
}

\author{
Feng Zeng \\ School of Software, Central South \\ University \\ Changsha, Hunan, China 410075 \\ fengzeng@csu.edu.cn
}

\author{
Wenjia Li \\ Department of Computer Science, \\ New York Institute of Technology \\ New York, New York, USA 10023 \\ wli20@nyit.edu
}

\begin{abstract}
In this paper, we propose an Energy-Balancing and SpectrumAware Clustering (EBSAC) algorithm for Cognitive Radio Sensor Network (CRSN). EBSAC does not require global Common Control Channel (CCC), and uses uneven clusters to achieve the energy balance of the network. Cluster radiuses are decided by the energy consumption of nodes. Cluster Heads (CHs) are selected according to the residual energy, channel condition including available channels and common channels with their upstream $\mathrm{CHs}$, and the distance to sink and their upstream CHs. When choosing cluster channels, we consider both the number of cluster members and the number of Relay Nodes (RNs) to increase connectivity between clusters. In addition, a routing algorithm without global CC$\mathrm{C}$ is designed for the CRSN. Performance evaluation reveals that the proposed algorithms are effective in term of balancing energy.
\end{abstract}

\section{CCS CONCEPTS}

- Networks $\rightarrow$ Network algorithms;

\section{KEYWORDS}

CRSN, energy balance, spectrum aware, global common control channel, unevenly clustering

ACM Reference format:

Xuehan Chen, Feng Zeng, and Wenjia Li. 2017. An Energy-Balancing and Spectrum-Aware Clustering Algorithm for Cognitive Radio Sensor Network. In Proceedings of ACM conference, Chongqing, China, July 2017 (MOBIMEDIA'17), 6 pages.

DOI: $10.475 / 123 \_4$

\section{INTRODUCTION}

Cognitive Radio (CR) is an intelligent wireless communication system, which enable the unlicensed Secondary Users (SUs) to sense for and operate in the underutilized spectrum

Permission to make digital or hard copies of all or part of this work for personal or classroom use is granted without fee provided that copies are not made or distributed for profit or commercial advantage and that copies bear this notice and the full citation on the first page. To copy otherwise, to republish, to post on servers or to redistribute to lists, requires prior specific permission and/or a fee.

Mobimedia 2017, July 13-14, Chongqing, People's Republic of China

Copyright (c) 2017 EAI 978-1-63190-156-0 owned by licensed or Primary Users (PUs) without causing unacceptable interference to the PUs activities. Network performance can be improved through effective utilization of spectrum resources. Wireless sensor nodes equipped with CRs have revealed a new network paradigm, which is called Cognitive Radio Sensor Network (CRSN) [1]. In a CRSN, sensor nodes can detect available channels by spectrum sensing and select communication channels by spectrum decision. When the primary users appear on their communication channels, they will detect the PUs immediately and switch to another available channel if necessary.

Since CRSN is the combination of WSN and CR, we need not only consider the energy constraint and hardware limitation inherited from the traditional WSN, but also overcome the spectrum sensing and spectrum management problem. As one of solutions to the above both considerations, clustering can balance the energy consumption in WSNs, and support cooperative of the CR operations, such as channel sensing and channel access. Clustering is a structured way to manage topology effectively and to increase the system capacity, network performance and stability effectively [2].

Recently, there are some works on the clustering algorith$\mathrm{m}$ in CRSNs [3-9]. In these works, the authors considered both energy and spectrum for the selecting of Cluster Heads (CHs) and Cluster Channels (CCs), and the processing of clustering, with attention to prolong the lifetime of the network. However, in the existing works, at least one global Common Control Channel (CCC) was required to exchange the clustering messages and transmit data among CHs. It is difficult to meet this requirement in practice, especially in large scale CRSNs.

In this paper, we focus on clustering algorithm and routing protocol in CRSNs with considerations of both energy balancing and spectrum allocation, and propose an EnergyBalancing and Spectrum-Aware Clustering (EBSAC) protocol, which does not require any global CCC.

The rest of this paper is organized as follows. Section 2 presents the network model and assumptions. The design of the proposed algorithm EBSAC and routing algorithm are discussed in Section 3 and Section 4 respectively. Section5 focuses on the performance evaluation of the proposed algorithm. Finally, a summary of our proposed methods and future works are discussed in Section 6. 


\section{RELATED WORK}

Recently, CRSN has attracted much research attentions in the world, and some literatures including clustering algorithms have been studied [3-9].

Shah et al. [3] proposed a spectrum-aware cluster-based routing (SCR) protocol for CRSN, which overcome the formidable limitations for both energy and spectrum. It was the first routing protocol for CRSN that addressed the issues of both energy and spectrum constraints. The selection of cluster head depended on the energy and available channel. SCR assumed there was at least one global CCC in the network to exchange clustering messages and transmit data among CHs. Based on SCR, Shah et al. [4] proposed a spectrum-aware cluster-based energy efficient multimedia (SCEEM) routing protocol for CRSNs. In SCEEM, the number of clusters was optimally determined to minimize the distortion in multimedia quality that occurred due to packet losses and latency.

Eletreby et al. [5] introduced Cognitive LEACH (CogLEACH) protocol, which was a spectrum-aware extension of the Low Energy Adaptive Clustering Hierarchy (LEACH) protocol. CogLEACH used the number of vacant channels as the weight for the probability of each node becoming a cluster head. It improved the throughput and lifetime of the network compared to the regular LEACH protocol. However, it did not consider energy balancing and required global CCC.

A distributed spectrum aware clustering (DSAC) algorith$\mathrm{m}$ was proposed in the literature as well [6]. DSAC derived the optimal number of clusters and minimized the communication energy. In DSAC, the clusters were organized such that the total communication power was minimized, and it considered the energy saving issue in intra-cluster data aggregation and inter-cluster relaying. However, in order to balance the energy consumption with a cluster, nodes in the cluster had the equal probability to become $\mathrm{CH}$.

Ozger et al. [7] proposed an Event-driven Spectrum-Aware Clustering algorithm named ESAC. ESAC formed temporal cluster for each event, and clusters were only formed between event and sink. During the process of clustering, ESAC considered both the number of available channels and the member nodes within two hops to achieve the best network connectivity. However, in ESAC, communication among nodes and $\mathrm{CH}$ was single-hop communication and ESAC confined to event-driven CRSN so that it could not efficiently operate in time-triggered CRSN scenario and other scenarios.

In literature [8], the authors proposed a low energy adaptive uneven clustering hierarchy for CRSN named LEAUCH. It not only considered the advantage of the channel resource in reducing the energy consumption but also applied uneven clustering method for balancing the energy consumption among the cluster heads in multiple hop communication. LEAUCH assumed that the entire network had a networkwide CCC and radiuses of clusters only depend on the distance between $\mathrm{CH}$ and sink.

An Energy-Efficient and Compact Clustering Scheme with Temporary Support Nodes for CRSN named CENTER was proposed in [9]. It introduced the concept of temporary support nodes to improve the cluster formation. Sensor nodes in CENTER can find a cluster head efficiently. However, temporary support nodes consumed much energy to help other nodes to find the corresponding cluster.

Although compared with most of the existing clustering algorithms, the above clustering algorithms can operate in CRSN more efficiently. However, the network-wide CCC is difficult to achieve in practice, and in order to prolong the lifetime of CRSN, we should balance the energy consumption throughout the network.

Based on the above discussion, we study the energy-balancing and spectrum-aware clustering algorithm in CRSNs. We propose EBSAC protocol, which does not need global CCC and considers both energy and spectrum when clustering. At last, theoretical analysis and simulation results show the effectiveness and superiority of the proposed algorithms.

\section{NETWORK MODEL AND ASSUMPTIONS}

In this section, we describe the network model and energy consumption model along with some assumptions.

\subsection{Network Model}

In this paper, we consider the network which is composed of $M$ Primary Users (PUs), $N$ Secondary Users (SUs) and a Sink Node $(\mathrm{SN})$. The set of PUs and SUs are defined as $P=\left\{p_{1}, p_{2}, \cdots, p_{M}\right\}$ and $S=\left\{s_{1}, s_{2}, \cdots, s_{N}\right\}$, respectively. There are $Q$ non-overlapping orthogonal channels in the network. The set of channels is represented as $C=$ $\left\{c_{1}, c_{2}, \cdots, c_{Q}\right\}$, in which $c_{i}$ represents the $i$-th channel. The PUs can utilize their licensed bands directly, and the SUs can detect the vacant channels by spectrum sensing and only use the channel that is not used by PUs in an opportunistic manner. Every node has its available channel set which is represented as $C_{i}$.

In this paper, we assume the process of channel sensing mechanism has finished and vacant spectrum bands do not change during clustering. Thus, the process of channel sensing is ignored in this paper. The SUs are stationary after the deployment is completed. Hence, neighbors do not change unless they are dead when their energy is exhausted. Each SU periodically senses the environment and generates data, all SUs in the network have the same data generation rate $\varphi$. Two SUs $\left(s_{i}\right.$ and $\left.s_{j}\right)$ can communicate with each other if they satisfy the following two constraints: $\varnothing$.

(1) They have the same available channels. That is $C_{i} \cap C_{j} \neq$

(2) They meet the communication distance constraint. That is $d_{i j} \leq R_{t}, d_{i j}$ is the distance between $s_{i}$ and $s_{j}$, and $R_{t}$ is the maximum communication distance.

\subsection{Energy Consumption Model}

In this paper, we assume that the initial energy of each node is $E_{i n i}$, and we use the energy consumption model described in Ref. [10]. Both the free space $\left(d^{2}\right.$ power loss) and the 
multi-path fading ( $d^{4}$ power loss) channel models are used in the energy consumption model, depending on the distance between the transmitter and receiver. The energy spent for transmission of a packet with $l$ bits size over distance $d$ is Equation 1.

$$
E_{\text {member }}= \begin{cases}l E_{\text {elec }}+l \varepsilon_{f s} d^{2}, & \text { if } d<d_{0} \\ l E_{\text {elec }}+l \varepsilon_{\text {amp }} d^{4}, & \text { if } d \geq d_{0}\end{cases}
$$

In Equation 1, $E_{\text {elec }}$ is the electronics energy, $\varepsilon_{f s}$ and $\varepsilon_{a m p}$ are the energy required by power amplification. The energy used to receive this message is $E_{R}(l)$ shown in Equation 2.

$$
E_{R}(l)=l E_{\text {elec }}
$$

It is assumed that the sensed information is highly correlated. Thus, the CHs can always aggregate the data gathered from their members into a single length-fixed packet. The rate of data aggregation is $\phi$, and we assume that a $\mathrm{CH}$ consumes $E_{d f}$ amounts of energy for data aggregation. In this paper, the above parameters are the same as the parameters used in Ref. [10].

\section{ENERGY-BALANCING AND SPECTRUM-AWARE CLUSTERING}

The main goal of our proposed Energy-Balancing and SpectrumAware Clustering(EBSAC) algorithm is to divide the CRSN into clusters, meanwhile ensuring the energy balancing and connectivity of CRSN in the case of no global CCC in the network. The intra-cluster and inter-cluster connectivity is a challenge as there is no global CCC. There are four types of role in the clustering infrastructure creation, described as follows.

(1) $\mathrm{SN}$, a node receiving data from the CHs.

(2) Cluster Header $(\mathrm{CH})$, a node that is responsible for gathering all data sent by its $\mathrm{CMs}$, aggregating them and sending the result toward the sink node.

(3) Cluster Member (CM), a node that reports the gathered data to its Cluster Header.

(4) Relay Node $(\mathrm{RN})$, a node that is a Cluster Member and assists Cluster Header to transmit data to sink.

The EBSAC algorithm has two key functions. The one is calculation of cluster radiuses of each node. In multi-hop network, the node near sink consumes more energy than the node far away from the sink. So in EBSAC, the uneven cluster is adopted to balance the energy consumption of the network.

The other key function is cluster construction. The cluster is constructed in three phases. (1) The first phase is the selection of CHs. As CHs has more energy consumption than other nodes and need to communicate with $\mathrm{CMs}$ and other $\mathrm{CHs}$, we select the $\mathrm{CH}$ s from all nodes of the network according to their residual energy, the number of available channels and the distance to sink or their upstream CHs. (2) The second phase is the determination of Cluster Channel (CC). As there is no global CCC in the network, we should ensure the connectivity of the network, including the intra-cluster and inter-cluster connectivity. In EBSAC, CC is determined depend on the number of CMs and RNs of the cluster. (3) The last phase is the establishment of the cluster. Every node belongs to only one cluster.

\subsection{Calculation of Cluster Radius}

In multi-hop network, nodes near the SN consume more energy than the nodes far away from the SN. Thus, nodes near the SN will die fast as soon as they exhaust their energy. In this paper, the lifetime of the network is defined as the time of death of the first node. In order to prolong the lifetime of CRSN, in EBSAC, uneven cluster is adopted to achieve the energy balancing throughout the network. In a cluster, there are some $\mathrm{CMs}$ and a $\mathrm{CH}$. $\mathrm{CMs}$ send their data to the $\mathrm{CH}$. $\mathrm{CHs}$ gather all data sent by their CMs, aggregate them and send the result toward their next hop to SN. The next hop of $\mathrm{CH}$ can be sink, another $\mathrm{CH}$ (its upstream $\mathrm{CH}$ ) and $\mathrm{RN}$, if the $\mathrm{CH}$ has $\mathrm{CCC}$ with its upstream $\mathrm{CH}$, its next hop is the upstream $\mathrm{CH}$, else if the distance between $\mathrm{CH}$ and sink larger than $R_{t}$, its next hop is one of RNs, otherwise, its next hop is the sink node. However, the nodes will send their data to sink directly if the distance between the node and sink is less than $r_{0}$, and its maximum energy consumption is $E_{0}$ in Equation 3.

$$
E_{0}=E_{e} l e c+\alpha\left(r_{0}\right)^{\beta} .
$$

In Equation 3, $\alpha$ and $\beta$ depend on $r_{0}$ according Equation 1.

It is assumed that the set of $\mathrm{CHs}$ without upstream $\mathrm{CH}$ is $C h_{1}$, all nodes in $C h_{1}$ will send their data to the sink directly. Let $s_{i} \in C h_{1}$, the energy consumption of $s_{i}$ is $E_{i}$ in Equation 4.

$$
E_{i}=E_{i}^{i n}+E_{i}^{o u t}
$$

In Equation $4, E_{i}^{\text {in }}$ and $E_{i}^{\text {out }}$ are the energy consumption used for intra-cluster and inter-cluster transmission respectively, and satisfy the Equations 5 and 6 , where $d_{i, s i n k}$ is the distance between $s_{i}$ and sink, $r_{i}$ is the cluster radius of $s_{i}$.

$$
\begin{gathered}
E_{i}^{i n}=\rho \pi r_{i}^{2}\left[(1+\phi) E_{\text {elec }}+E_{d f}+\phi \alpha d_{i, \text { sink }}^{\beta}\right] \\
E_{i}^{\text {out }}=\frac{\phi \rho \pi r_{i}\left[R^{2}-\left(r_{0}+2 r_{i}\right)^{2}\right]\left(2 E_{\text {elec }}+\alpha d_{i, \text { sink }}^{\beta}\right)}{4\left(r_{0}+r_{i}\right)}
\end{gathered}
$$

It is assumed that $C h_{x}$ is the set of CHs having upstream $\mathrm{CH}$. Then, all nodes in $C h_{x}$ will send their data to their upstream CHs. Let $s_{j} \in C h_{x}$, its energy consumption is $E_{j}=E_{j}^{\text {in }}+E_{j}^{\text {out }}$, and $E_{j}^{\text {in }}$ and $E_{j}^{\text {out }}$ are shown in Equations 7 and 8 respectively. In the two equations, $d_{j}^{c}$ is the distance between $s_{j}$ and its upstream $\mathrm{CH}$ (or RN), and $r_{j}$ is the cluster radius of $s_{j}$.

$$
\begin{gathered}
E_{j}^{i n}=\rho \pi r_{j}^{2}\left[(1+\phi) E_{\text {elec }}+E_{d f}+\phi \alpha\left(d_{j}^{c}\right)^{\beta}\right] \\
E_{j}^{\text {out }}=\frac{\phi \rho \pi r_{j}\left[R^{2}-\left(d_{j, \text { sink }}+r_{j}\right)^{2}\right]\left(2 E_{\text {elec }}+\alpha\left(d_{j}^{c}\right)^{\beta}\right)}{4 d_{j, \text { sink }}}
\end{gathered}
$$


In order to balance the energy consumption of the network, we should have $E_{0}=E_{i}$ and $E_{0}=E_{j}$. We can get $d_{j, \text { sink }}$ and $d_{j}^{c}$ through GPS or other technology. For a given $r_{0}$, we can get the corresponding $r_{i}$ and $r_{j}$ satisfying $E_{0}=E_{i}$ and $E_{0}=E_{j}$ respectively.

\subsection{Cluster Construction}

The main goal of the cluster construction is to divide to network into several clusters. So first we need to select some nodes as $\mathrm{CHs}$, then assign the clusters with proper channels, and finish the clusters construction finally.

\subsubsection{Selection of $\mathrm{CHs}$}

. In clustering-based CRSN, we need to consider the energy problem inherited from the traditional WSN, and address the channel problem inherited from CRN. As there is no global CCC in the network, we should ensure the connectivity of the network during the process of clustering.

In EBSAC, we choose the $\mathrm{CHs}$ based on the following three aspects: residual energy, the number of available channels and the distance to sink and upstream $\mathrm{CH}$. The best option should have:

(1) More residual energy. As CH consumes more energy than other nodes, in order to balance the energy consumption throughout the network, the $\mathrm{CH}$ should have more residual energy.

(2) More available channels. As there is no global CCC, in order to ensure the connectivity of intra-cluster and intercluster, $\mathrm{CH}$ should have more available channels so that it can communicate with its CMs, other CHs and RNs. The more available channels of CHs, the more successful the clustering process.

(3) Near the sink. The CHs need to transmit the data toward the SN. Reducing the distance between $\mathrm{CH}$ and sink can reduce the energy consumption of $\mathrm{CH}$.

Based on the above discussion, we define Node Value as the metric to choose the CHs.

Define 1 (Node Value, NV): NV represents the degree of the node for data communication. It reflects the energy, available channels and distance situation of the node and is the most important metric to determine CHs. It can be defined as Equations 9, 10, 11 and 12.

$$
\begin{gathered}
N V(i)=\alpha f(E)+\beta g(C)+\gamma h(D) \\
f(E)=\frac{E_{i}^{\text {res }}}{E_{\text {ini }}} \\
g(C)=\frac{\left|C_{i}\right|+\left|C_{C}\right|}{|C|} \\
h(D)=\left(1-\frac{d_{i, \text { sink }}}{R}\right)\left(1-\frac{d_{i}^{c}}{R}\right)
\end{gathered}
$$

In the above Equations 9, 10, 11 and 12, $N V(i)$ is the node value of node $s_{i}, f(E), g(C)$ and $h(D)$ are the functions of energy, channel and distance respectively. The parameters $\alpha, \beta$ and $\gamma$ are the coefficient factor of $f(E), g(C)$ and $h(D)$ respectively, while satisfying $\alpha+\beta+\gamma=1$. $\left|C_{c}\right|$ is the number of common channels of node $s_{i}$ with its upstream $\mathrm{CH}$.

We can know from the definition of NV, during the selection of CHs, we consider three aspects simultaneously: the residual energy of the node, channel condition (both the available channel of the node and the common channel with its upstream $\mathrm{CH}$ ) and the distance to the sink and its upstream $\mathrm{CH}$.

Every node needs to send its information to sink, and the information includes: residual energy, available channel and position. The process of selection of the $\mathrm{CHs}$ is completed by $\mathrm{SN}$, thus the energy consumption of the node is reduced. $\mathrm{SN}$ determines $\mathrm{CH}$ s from the inside out: each time selects the node which has the greatest $\mathrm{NV}$ as $\mathrm{CH}$.

\subsubsection{Determination of Cluster Channel}

. Once a SU is selected as $\mathrm{CH}$, it wants to select the cluster channel and then form the cluster, and so on. In EBSAC, as there is no global CCC, cluster channel has an important impact on the inter-cluster and inter-cluster connectivity, and the efficiency of clustering. Cluster channel decides the CMs of cluster. In order to make sure the connectivity of the network (both intra-cluster and inter-cluster), and improve the efficiency of clustering, we choose the cluster channel based on the number of CMs and RNs. A good cluster channel should satisfy:

(1) Making the cluster has more CMs.

(2) Making the cluster can communicate with its upstream cluster.

Based on the above discussion, in this paper, Channel Value is defined, and used to choose the cluster channel.

Define 2(Channel Value, CV): CV represents the worth of the channel, and is the main metric to choose the cluster channel. It can be defined as Equation 13.

$$
C V_{i}= \begin{cases}\left|N M_{i}\right|, & \text { if } C_{i} \text { is } C C \\ \left|N M_{i}\right| \times\left|N N_{i}\right|, & \text { otherwise }\end{cases}
$$

In Equation 13, $\left|N M_{i}\right|$ is the number of CMs when the cluster channel is $c_{i}$, and $\left|N N_{i}\right|$ is the number of RNs (the nodes in the relay region as shown in Fig. 1) of cluster with cluster channel $c_{i}$.

In EBSAC, cluster channel is selected depending on the value of $C V$. We select the channel with the maximum value of CV as cluster channel.

\subsubsection{Cluster Establishment}

. Once the processes of selection of $\mathrm{CH}$ and determination of cluster channel are finished, the cluster establishment will begin. It is assumed $s_{m}$ is the $\mathrm{CH}$ of cluster $\mathrm{H}$, and the corresponding cluster channel and cluster radius are $c_{n}$ and $r_{m}$ respectively. Node $s_{i}\left(s_{i} \in S\right)$ is one of the CM of cluster $\mathrm{H}$ should satisfy the following constraints:

(1) It does not belong to any other cluster. Every node of the network can belong to only one cluster.

(2) Channel $c_{n}$ is one of available channel of $s_{i}$.

(3) The distance between $s_{m}$ and $s_{i}$ is less than or equal to the cluster radius $r_{m}$, that is $d_{s_{m}, s_{i}} \leq r_{m}$. 
During the process of cluster establishment, the $\mathrm{CH}$ broadcasts the Cluster Establishment Message (CEM), the CEM includes the coordinate of the $\mathrm{CH}$. The node which receives CEM and satisfies the above constraints becomes the CM of the cluster.

\section{ROUTING}

As there is no global CCC in the network, the existing routing algorithms cannot efficiently operate in the network. In this paper, we design a routing algorithm can efficiently operate in the network which has no global CCC. During the routing, we consider not only the distance between transmitter and receiver, but also the CCC of transmitter and receiver.

The proposed algorithm considers the following four types of role in the routing infrastructure creation: $\mathrm{SN}, \mathrm{CM}, \mathrm{CH}$ and RN. The responsibility of each role has been discussed in section 4. The main goal of routing is to find the next hop of each node until the next hop is SN. Next we discuss the next hop of each role in detailed.

(1) The next hop of the nodes having distance to sink is less than $r_{0}$.

In order to reduce the energy consumption of the network, similar to the existing routing algorithms, if the distance between the node and sink is less than $r_{0}$, it sends its data to SN directly.

(2) The next hop of CM.

Every CM belongs to only one cluster. The CMs need to report the gathered data to its corresponding $\mathrm{CH}$. For a $\mathrm{CM}$, if the distance between the $\mathrm{CM}$ and the $\mathrm{CH}$ is less than or equal to the maximum transmission range $R_{t}$, it sends its data to the $\mathrm{CH}$ directly. Otherwise it transmits its data to the $\mathrm{CH}$ through the relay of RNs. As there is at least one CCC in the cluster (i.e. cluster channel, we establish the cluster according the cluster channel and cluster radius), so we choose the RNs only depend on the residual energy and the distance between $\mathrm{CH}$ and RNs [11].

(3) The next hop of $\mathrm{CH}$.

In the paper, the most important part of routing is the selection of the next hop of $\mathrm{CH}$. The $\mathrm{CH}$ is responsible for gathering all the gathered data sent by its CMs, aggregating them and sending the result toward the sink node. As there is no global CCC in the network, so the common channel of inter-cluster has an important impact on routing. In EBSAC, during the process of selection of $\mathrm{CH}$, we take the available channel of $\mathrm{CH}$ into consideration. During the determination of cluster channel, we both consider the number of CMs and RNs which have the available channel of cluster channel.

Thus, we choose the next hop of $\mathrm{CH}$ based on the following rules: if the $\mathrm{CH}$ has the common channel with its upstream $\mathrm{CH}$, it transfers the data to its upstream $\mathrm{CH}$ directly, as shown in Fig. 1 (Path1). Otherwise, they need the relay of $\mathrm{RN}$ to transmit the data to its upstream $\mathrm{CH}$, as shown in Fig. 1 (Path2).

(4) The next hop of RN. As the RN is a subset of the CM, so the next hop of $\mathrm{RN}$ is same to the CM.

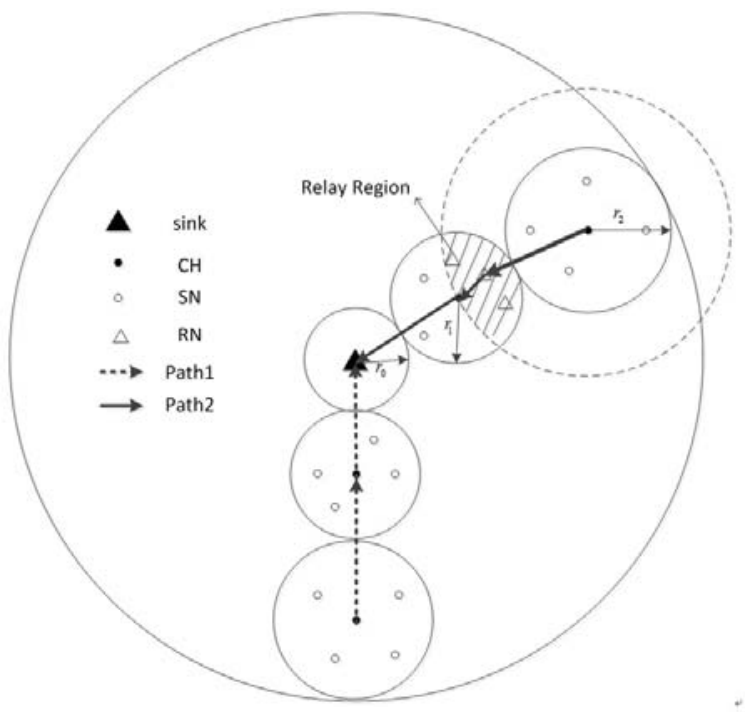

Figure 1: Routing path.

\section{PERFORMANCE EVALUATION}

In this section, the effectiveness of the proposed protocol is evaluated via simulation experiments. The proposed algorithm is simulated in OMNet++, and effectiveness and performance of the algorithm are analyzed on the basis of the simulation results. SN is located at the center of the network $(0,0)$, and there are 5 PUs and 500 SUs in the network. The radius of the network is 200 meters. The number of channel in the network is 8 . Each sensor periodically senses the environment and generates data, the generation rate is 0.2 .

\subsection{Network Lifetime}

As WSN is resource-constraint, nodes are dead when their energy is exhausted. It is very important to prolong the lifetime of the network. With the increasing rounds of data transmission, the number of living nodes of the network is shown in Fig. 2. From Fig. 2, we can clearly find that the lifetime of CRSN achieved by EBSAC is similar to the lifetime of CRSN achieved by LEAUCH [8]. But, compared with LEAUCH, EBSAC has no global CCC.

\subsection{Energy Balance}

Fig. 3 and 4 show the residual energy of nodes when the first node dies in EBSAC and LEAUCH respectively. We can find that EBSAC has the better energy balance compared with LEAUCH. The CHs near the sink have higher energy consumption than the CHs far away from the sink. In EBSAC, the energy consumption of the $\mathrm{CH}$ is relatively balanced.

\section{CONCLUSION}

In this paper, an Energy-Balancing and Spectrum-Aware Clustering (EBSAC) is proposed for CRSN with no global CCC. As there is no global CCC in the network, so the 


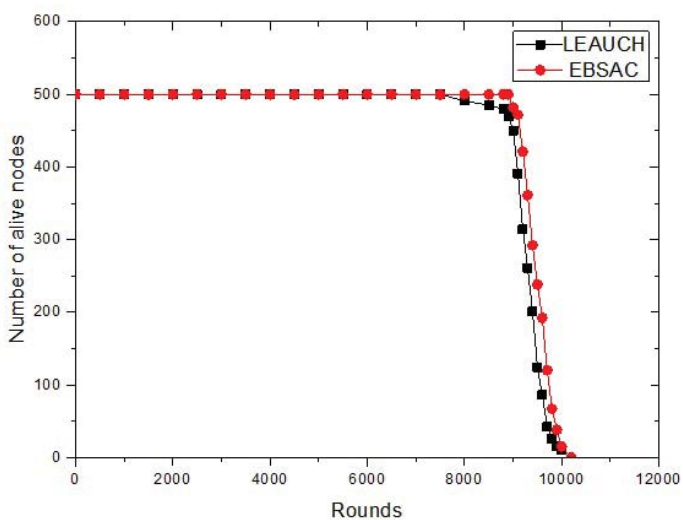

Figure 2: Network lifetime comparison.

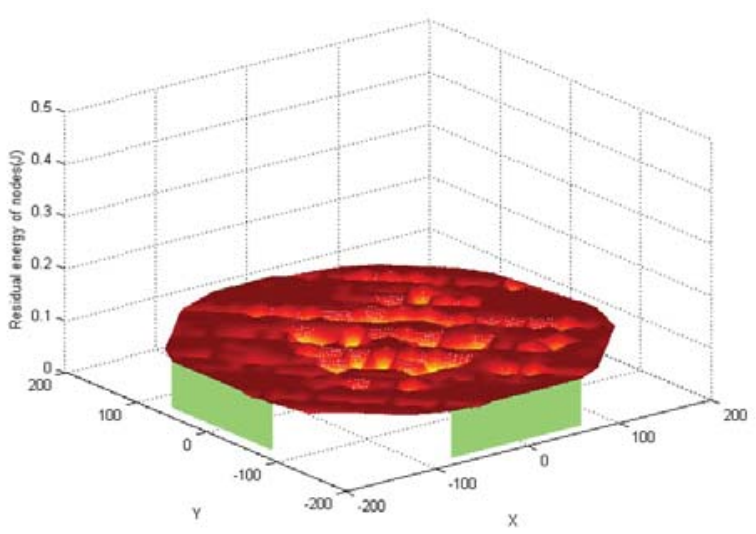

Figure 3: Residual energy of nodes in EBSAC.

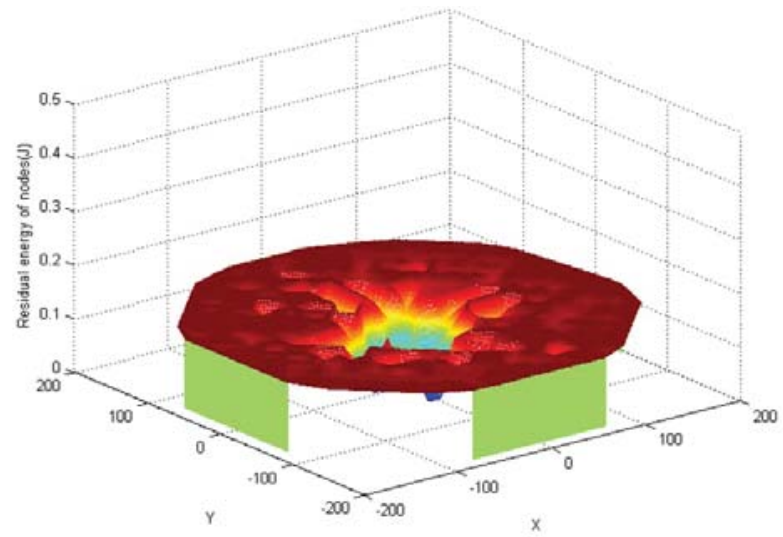

Figure 4: Residual energy of nodes in LEAUCH.

existing clustering and routing algorithms cant operate effectively. In EBSAC, we take both the energy balance and spectrum allocation into consideration. In order to achieve the energy balance of the network, the uneven-cluster is used in this paper, and the cluster radius is determined depends on the actual energy consumption. During the routing, the selection of the next hop also considers the energy factor. In order to ensure the inter-cluster and intra-cluster connectivity, during the process of cluster construction, we not only consider the common channel between $\mathrm{CMs}$ and $\mathrm{CH}$ in the cluster, but also take the common channel between $\mathrm{CHs}$ into consideration. In addition, a routing algorithm based on EBSAC is designed to overcome the constraints of having no global CCC. Performance evaluation shows that the proposed algorithms are energy-efficient even if there is no CCC in the network.

Acknowledgements: This work is supported in part by the National Natural Science Foundation of China (61672540, 61379057) and Teacher Research Fund in Central South University (2014JSJJ019).

\section{REFERENCES}

[1] Ozgur B. Akan, Osman B. Karli, and Ozgur Ergul. Cognitive radio sensor networks. IEEE Network, 23(4):34-40, August 2009.

[2] J. Y. Yu and P. H. J. Chong. A survey of clustering schemes for mobile ad hoc networks. IEEE Communications Surveys and Tutorials, 7(1):32-48, January 2005.

[3] G Shah and O B Akan. Spectrum-aware cluster-based routing for cognitive radio sensor networks. In ICC'13 Conference Proceedings, pages 2885-2889. IEEE, June 2013.

[4] Ghalib A. Shah, Fatih Alagoz, Etimad A. Fadel, and Ozgur B. Akan. A spectrum-aware clustering for efficient multimedia routing in cognitive radio sensor networks. IEEE Transactions on Vehicular Technology, 63(7):3369-3380, July 2014.

[5] R M Eletreby, H M Elsayed, and M M Khairy. A spectrum aware clustering protocol for cognitive radio sensor networks. In CROWNCOM'14 Conference Proceedings, pages 179-184. EAI, June 2014.

[6] Huazi Zhang, Zhaoyang Zhang, Huaiyu Dai, Rui Yin, and Xiaoming Chen. Distributed spectrum-aware clustering in cognitive radio sensor networks. In GLOBECOM'11 Conference Proceedings, pages 1-6. IEEE, December 2011.

[7] M Ozger and O B. Akan. Event-driven spectrum-aware clustering in cognitive radio sensor networks. In INFOCOM'13 Conference Proceedings, pages 1483-1491. IEEE, April 2013.

[8] Errong Pei, Haozhe Han, Zehua Sun, Bin Shen, and Tianqi Zhang. Leauch: Low-energy adaptive uneven clustering hierarchy for cognitive radio sensor network. EURASIP Journal on Wireless Communications and Networking, 2015(1):1-8, April 2015.

[9] Shelly Salim, Sangman Moh, Dongmin Choi, and Ilyong Chung. An energy-efficient and compact clustering scheme with temporary support nodes for cognitive radio sensor networks. Sensors, 14(8):14634-14653, August 2014

[10] X.B. Wu, G. Chen, and Sajal K. Das. Avoiding energy holes in wireless sensor networks with non-uniform node distribution. IEEE Transactions on Parallel and Distributed Systems, 19(5):710C720, August 2008.

[11] L A Villas, A Boukerche, H S Ramos, H F Oliveira, R B Araujo, and A F Loureiro. Drina: A lightweight and reliable routing approach for in-network aggregation in wireless sensor networks. IEEE Transactions on Computers, 62(4):676-689, April 2013. 